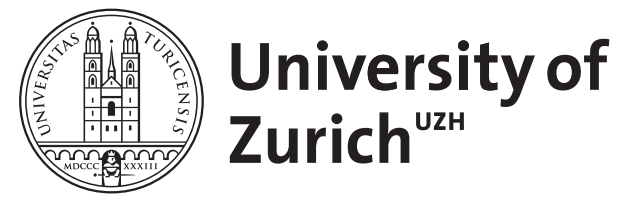

Zurich Open Repository and Archive

University of Zurich

University Library

Strickhofstrasse 39

CH-8057 Zurich

www.zora.uzh.ch

Year: 2013

Le chat du docteur Frankenstein : modifications biotechnologiques de nos amis les bêtes

Roduit, Johann A R

Posted at the Zurich Open Repository and Archive, University of Zurich

ZORA URL: https://doi.org/10.5167/uzh-79935

Journal Article

Published Version

Originally published at:

Roduit, Johann A R (2013). Le chat du docteur Frankenstein : modifications biotechnologiques de nos amis les bêtes. Schweizerische Ärztezeitung (SÄZ), 94(33):1232-1233. 


\section{Le chat du docteur Frankenstein: modifications biotechnologiques de nos amis les bêtes}

\section{Johann Roduit}

Doctorant en droit et éthique biomédicale à l'Institut d'éthique biomédicale de l'Université de Zurich, Cofondateur du think tank NeoHumanitas.
Correspondance: Johann Roduit Institute of Biomedical Ethics Pestalozzistrasse 24 CH-8032 Zurich Tél. 0796653551

johann.roduit[at]ethik.uzh.ch www.neohumanitas.org
Souris marathoniennes, poissons transgéniques, insectes cyborgs... les animaux que l'on trouve dans les œuvres de science-fiction envahissent gentiment notre quotidien. Dans Frankenstein's Cat: Cuddling up to Bioetech's Brave New Beasts, la journaliste américaine Emily Anthes dresse un tour d'horizon des transformations possibles faites sur les animaux grâce aux nouvelles technologies.

Selon cette auteure, la façon dont nous traitons les animaux est ambiguë. D'une part, nous souhaitons les protéger et améliorer leur existence. D'autre part, nous ne cessons de les instrumentaliser pour nos recherches et pour nous nourrir. Néanmoins, Anthes estime que les nouvelles technologies utilisées pour modifier les animaux ne sont pas intrinsèquement bonnes ou mauvaises: nous avons le choix d'y recourir - ou non - dans le but d'améliorer la vie de tout un chacun.

Son livre, composé de huit chapitres, peut être divisé en trois parties distinctes. La première partie (chapitres 1-2) se focalise sur les modifications génétiques utilisées à des fins commerciales, esthétiques ou scientifiques. Elle s'attarde notamment sur certains poissons transgéniques et phosphorescents qui décorent d'ores et déjà l'aquarium de certains Américains, ainsi que sur des moutons génétiquement transformés qui produisent du lait modifié utilisé pour créer de nouveaux médicaments. En deuxième partie (ch. 3-4), Anthes se concentre sur le clonage. Cette technique permet déjà de répliquer un animal de compagnie décédé. D'autres voient dans le clonage une possibilité de protéger les espèces en danger, ou même de redonner vie à des espèces disparues. Les trois chapitres suivants (ch. 5-7) décrivent les modifications technologiques utilisées à des fins scientifiques, thérapeutiques, ou militaires. Certains animaux sont, en effet, équipés de capteurs (radio ou wifi) qui nous permettent de les suivre et de les étudier quotidiennement. Certains ingénieurs proposent également des traitements animaliers qui consistent en la création de prothèses artificielles sur mesure pour les animaux qui présenteraient des problèmes articulaires (paralysie, arthrose, etc.). Enfin, certains insectes peuvent être transformés en «cyborgs espions» à des fins militaires. C'est dans le dernier chapitre (ch. 8) que la thèse principale du livre apparaît: l'être humain doit prendre ses responsabilités et accepter son rôle en tant que force dominante pour l'avenir de la planète. Il doit (re)découvrir ce que signifie «être gardien de la création». Dès lors

\section{FRANKENSTEIN'S CAT}

\section{CUDDLING UP TO BIOTECH'S BRAVE NEW BEASTS \\ EMILY ANTHES}

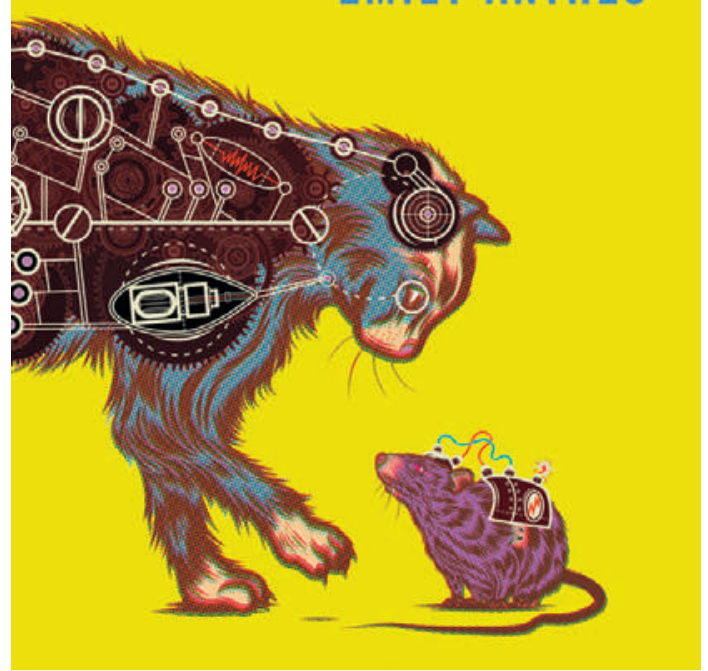

Emily Anthes. Frankenstein's Cat. Cuddling up to Biotech's Brave New Beasts. New York: Scientific American / Farrar, Straus and Giroux; 2013. 256 pages, hardcover, 26 USD. ISBN 978-0-3741-5859-0

qu'Homo sapiens continue d'améliorer ses capacités au-delà de la thérapie, Anthes l'appelle à ne pas oublier d'améliorer également les capacités des animaux, afin qu'ils puissent évoluer parallèlement.

En plus de l'excellent travail de recherche journalistique, Anthes pose d'intéressantes questions éthiques et géopolitiques. Tout au long de son ouvrage, l'auteure soulève des questions éthiques relatives à la santé et à l'autonomie des animaux. Elle dénonce l'instrumentalisation et l'exploitation de ces derniers. Elle met en garde contre une certaine arrogance de l'être humain qui veut tout contrôler sans un consentement «véritable» des animaux. En citant le biologiste Marc Bekoff, Anthes nous rappelle que «Les animaux ont les mêmes désirs que les êtres humains. Ils veulent éviter la douleur, ils veulent juste être contents, ils veulent que leurs besoins de nourriture, de logement, d'amitié, de sexe ou de quoi que ce soit soient satisfaits, et ils veulent 
éviter les souffrances, l'inconfort, le stress et la terreur» $(71)^{1}$. Anthes suggère donc de suivre le principe défendu par le philosophe Bernard Rollin: «Si vous modifiez des animaux, les animaux qui en résultent ne doivent pas être plus mal d'un point de vue du bien-être, ils devraient même être mieux qu'avant, de préférence» (49-50).

L'utilisation de ces nouvelles technologies pose aussi des problèmes géopolitiques. «Si d'autres nations commencent à approuver, et peut-être exporter un produit animal transgénique, elles vont mettre la pression sur les Etats-Unis, le Canada et d'autres pays afin que ceux-ci acceptent également davantage d'organismes génétiquement modifiés» (54). Certains scientifiques ont déjà déplacé leurs laboratoires au Brésil, en Argentine, en Inde ou en devons le faire - avec quels outils, dans quelles circonstances et dans quel but» (177). Elle pose donc des questions intéressantes en guise de conclusion: «Et si nous remplacions les jambes vieillissantes des chiens avec des prothèses qui leur permettent de courir plus vite et plus loin?» (180). Avec des jambes bioniques, par exemple, «Nous pourrions aider les animaux blessés ou âgés non seulement en les soignant, mais également en les transformant même mieux que la nature en avait eu l'intention» (180).

Les rencontres et les anecdotes personnelles, relatées tout au long de ce livre, donnent vie à cette œuvre. Elles nous permettent de parcourir le monde et de rencontrer ces animaux génétiquement modifiés ou clonés, ainsi que ces scientifiques, ces entrepreneurs, ces consommateurs et ces philosophes qui,

\section{Le débat sur la protection des animaux a déjà été amorcé. Celui sur l'amélioration animale au-delà de la thérapie ne fait que commencer.}

1 Toutes les citations de ce manuscrit sont des traductions personnelles.
Chine où les lois et règlements sont plus laxistes en matière de protection animale et où ils sont ainsi en mesure de développer leurs expériences.

L'auteure expose finalement un débat souvent négligé: les problèmes socio-éthiques soulevés par l'utilisation de certaines technologies dans le but «d'améliorer» les animaux (animal uplift), un débat qui s'inscrit dans un champ plus large qu'est celui de l'amélioration humaine (human enhancement). Simplement dit, la biotechnologie devrait nous permettre de bientôt pouvoir améliorer la vie des animaux au-delà de la thérapie. Anthes appelle l'être humain à prendre ses responsabilités en tant que force directrice de l'évolution de notre monde, afin de créer un monde amélioré, où les humains qui se perfectionnent, améliorent avec eux le monde animal et végétal. Pour l'auteure, «la vraie question, à l'avenir, n'est pas de savoir si nous devons façonner le corps et la vie des animaux, mais comment nous d'une manière ou d'une autre, influencent la vie de ces animaux modifiés.

Cet ouvrage pourrait séduire non seulement les personnes peu informées sur le sujet, mais également les spécialistes qui trouveront regroupées un grand nombre d'anecdote. Cette œuvre va au-delà de la simple description et pousse le lecteur à la réflexion en lui proposant quelques outils normatifs. Elle lance également le débat sur l'amélioration animale, un sujet encore très peu connu et débattu dans le milieu académique et dans le grand public.

Frankenstein's Cat devrait être apprécié par les amis des animaux, mais également par les scientifiques, les entrepreneurs, les philosophes, et plus généralement par tous ceux qui se soucient de notre futur. Le débat sur la protection des animaux a déjà été amorcé. Celui sur l'amélioration animale au-delà de la thérapie ne fait que commencer. 